\title{
Higgs Transverse Momentum with a Jet Veto: A Double-Differential Resummation
}

\author{
Pier Francesco Monni, ${ }^{1}$ Luca Rottoli $\odot{ }^{2}$ and Paolo Torrielli $\oplus^{3}$ \\ ${ }^{1}$ CERN, Theoretical Physics Department, CH-1211 Geneva 23, Switzerland \\ ${ }^{2}$ Dipartimento di Fisica G. Occhialini, U2, Università degli Studi di Milano-Bicocca and INFN, \\ Sezione di Milano-Bicocca, Piazza della Scienza, 3, 20126 Milano, Italy \\ ${ }^{3}$ Dipartimento di Fisica and Arnold-Regge Center, Università di Torino and INFN, Sezione di Torino, \\ Via P. Giuria 1, I-10125 Turin, Italy
}

(Received 26 September 2019; revised manuscript received 2 March 2020; accepted 22 May 2020; published 26 June 2020; corrected 8 February 2021)

\begin{abstract}
We consider the simultaneous measurement of the Higgs $\left(p_{t}^{H}\right)$ and the leading jet $\left(p_{t}^{J}\right)$ transverse momentum in hadronic Higgs-boson production, and perform the resummation of the large logarithmic corrections that originate in the limit $p_{t}^{H}, p_{t}^{J} \ll m_{H}$ up to next-to-next-to-leading-logarithmic order. This work constitutes the first simultaneous (double differential) resummation for two kinematic observables of which one involves a jet algorithm in hadronic collisions, and provides an important milestone in the theoretical understanding of joint resummations. As an application, we provide precise predictions for the Higgs transverse-momentum distribution with a veto $p_{t}^{J} \leq p_{t}^{J, v}$ on the accompanying jets, whose accurate description is relevant to the Higgs precision programme at the Large Hadron Collider.
\end{abstract}

DOI: 10.1103/PhysRevLett.124.252001

The thorough scrutiny of the properties of the Higgs boson [1,2] is central to the future physics program of the Large Hadron Collider (LHC). In the high-luminosity run of the LHC, the experimental precision in Higgs-related measurements will increase significantly [3], hence allowing for detailed studies of the Higgs sector of the standard model (SM) Lagrangian.

A full exploitation of such measurements requires an unprecedented level of precision in the theoretical description of the relevant observables. In this context, a prominent role is played by kinematic distributions of the Higgs boson and the accompanying QCD radiation, which are sensitive to potential new-physics effects, such as modifications of light-quark Yukawa couplings [4,5], or heavy new-physics states [6-11]. Experimental analyses of Higgs processes typically categorize the collected events in jet bins, according to the different number of jets-collimated bunches of hadrons in the final state-produced in association with the Higgs boson. Since the future performance of the LHC will allow for the precise measurement of kinematic distributions in different jet bins, it is paramount to achieve an accurate theoretical understanding of Higgs observables at the multidifferential level.

In this Letter we consider Higgs-boson production in gluon fusion, the dominant channel at the LHC, and we

Published by the American Physical Society under the terms of the Creative Commons Attribution 4.0 International license. Further distribution of this work must maintain attribution to the author(s) and the published article's title, journal citation, and DOI. Funded by SCOAP. focus on the Higgs transverse-momentum $\left(p_{t}^{H}\right)$ spectrum in the presence of a veto $p_{t}^{J, v}$ bounding the transverse momentum $p_{t}^{J}$ of the hardest accompanying jet. Veto constraints of such a kind are customarily enforced to enhance the Higgs signal with respect to its backgrounds, relevant examples being the selection of $H \rightarrow W^{+} W^{-}$ events from $t \bar{t} \rightarrow W^{+} W^{-} b \bar{b}$ production $[12,13]$ or the categorization in terms of different initial states [14].

Fixed-order perturbative predictions of the $p_{t}^{H}$ spectrum in gluon fusion are currently available at next-to-next-toleading order (NNLO) in the strong coupling $\alpha_{s}$ [15-19] in the infinite top-mass limit, and heavy-quark mass effects are known up to next-to-leading order (NLO) [20-24]. Fixed-order perturbation theory is, however, insufficient to accurately describe the observable considered here. When exclusive cuts on radiation are applied, it is well known that the convergence of the perturbative expansion is spoiled by the presence of logarithms $\ell \in\left\{\ln \left(m_{H} / p_{t}^{H}\right), \ln \left(m_{H} / p_{t}^{J, v}\right)\right\}$ that become large in the limit $p_{t}^{H}, p_{t}^{J, v} \ll m_{H}$, where the Higgs mass $m_{H}$ represents the typical hard scale of the considered process. In this regime, such large logarithmic terms must be summed to all perturbative orders to obtain a reliable theoretical prediction. The resummation accuracy is commonly defined at the level of the logarithm of the cumulative cross section, where terms of order $\alpha_{s}^{n} \ell^{n+1}$ are referred to as leading logarithms (LL), $\alpha_{s}^{n} \ell^{n}$ as next-toleading logarithms (NLL), $\alpha_{s}^{n} \ell^{n-1}$ as next-to-next-to-leading logarithms (NNLL), and so on. The resummation of the inclusive $p_{t}^{H}$ spectrum has been carried out up to high perturbative accuracy [25-28] and is currently known to $\mathrm{N}^{3} \mathrm{LL}$ order $[29,30]$. Such calculations have been combined 
with NNLO fixed order in Refs. [29-31] to obtain an accurate prediction across the whole $p_{t}^{H}$ spectrum. Similarly, the resummation of the jet-vetoed cross section has been achieved in Refs. [32-38], reaching NNLL accuracy matched to $\mathrm{N}^{3} \mathrm{LO}$ [39]. Related resummations of the transverse momentum imbalance of the Higgs and the hardest jet have been also considered in Refs. [40-42].

In this work, we present the first joint resummation of both classes of logarithms, by obtaining a prediction which is differential in both $p_{t}^{H}$ and $p_{t}^{J}$, and NNLL accurate in the limit $p_{t}^{H}, p_{t}^{J} \ll m_{H}$. Specifically, we integrate the double-differential distribution $d \sigma / d p_{t}^{J} d p_{t}^{H}$ over $p_{t}^{J}$ up to $p_{t}^{J}=p_{t}^{J, v}$, which results in the single-differential $p_{t}^{H}$ distribution with a jet veto. The results presented here are of phenomenological relevance in the context of the Higgs physics program at the LHC, and constitute an important milestone in the theoretical understanding of the structure of resummations of pairs of kinematic observables, which has received increasing interest lately [43-45]. Different kinds of joint resummations for hadronic Higgs production have been considered in the literature. Relevant examples are combined resummations of logarithms of $p_{t}^{H}$ and small $x$ [46,47], of $p_{t}^{H}$ and large $x$ [48-51], of small $x$ and large $x$ [52], and of $p_{t}^{J, v}$ and the jet radius [39].

To derive the main result of this Letter, it is instructive to first consider the standard transverse-momentum resummation [53,54], starting with a description of the effects that enter at NLL in a toy model with scale-independent parton densities. The core of the inclusive $p_{t}^{H}$ resummation lies in the description of soft, collinear radiation emitted off the initial-state gluons and strongly ordered in angle. Observing that in such kinematic configurations each emission is independent of the others, one obtains the following formula in impact-parameter $(b)$ space:

$$
\begin{aligned}
\frac{d \sigma}{d^{2} \vec{p}_{t}^{H}}= & \sigma_{0} \int \frac{d^{2} \vec{b}}{4 \pi^{2}} e^{-i \vec{b} \cdot \vec{p}_{t}^{H}} \\
& \times \sum_{n=0}^{\infty} \frac{1}{n !} \prod_{i=1}^{n} \int\left[d k_{i}\right] M^{2}\left(k_{i}\right)\left(e^{i \vec{b} \cdot \vec{k}_{t, i}}-1\right),
\end{aligned}
$$

where $\sigma_{0}$ denotes the Born cross section, and $\left[d k_{i}\right] M^{2}\left(k_{i}\right)$ is the phase space and squared amplitude for emitting a parton of momentum $k_{i}$. The exponential factor in Eq. (1) encodes in a factorized form the kinematic constraint $\delta^{2}\left(\vec{p}_{t}^{H}-\sum_{i=1}^{n} \vec{k}_{t, i}\right)$, while the -1 term in the round brackets arises because, by unitarity, virtual corrections come with a weight opposite to that of the real emissions, but do not contribute to $p_{t}^{H}$. The factorization of the phasespace constraint allows for an exact exponentiation of the radiation in Eq. (1), leading to the well-known formula of Refs. [53,54].

In order to include the constraint due to a veto on accompanying jets, let us first consider the effect of a jet algorithm belonging to the $k_{t}$-type family (such as the anti- $k_{t}$ algorithm [55]). Owing to the strong angular separation between the emissions, the clustering procedure at NLL will assign each emission to a different jet [32]. Therefore, imposing a veto $p_{t}^{J, v}$ on the resulting jets corresponds to constraining the real radiation with an extra factor

$$
\Theta\left(p_{t}^{J, v}-\max \left\{k_{t, 1}, \ldots, k_{t, n}\right\}\right)=\prod_{i=1}^{n} \Theta\left(p_{t}^{J, v}-k_{t, i}\right) .
$$

Plugging the above equation into Eq. (1) leads to

$$
\begin{aligned}
\frac{d \sigma\left(p_{t}^{J, v}\right)}{d^{2} \vec{p}_{t}^{H}} & =\sigma_{0} \int \frac{d^{2} \vec{b}}{4 \pi^{2}} e^{-i \vec{b} \cdot \vec{p}_{t}^{H}} \\
& \times \sum_{n=0}^{\infty} \frac{1}{n !} \prod_{i=1}^{n} \int\left[d k_{i}\right] M^{2}\left(k_{i}\right)\left[e^{i \vec{b} \cdot \vec{k}_{t, i}} \Theta\left(p_{t}^{J, v}-k_{t, i}\right)-1\right] \\
& =\sigma_{0} \int \frac{d^{2} \vec{b}}{4 \pi^{2}} e^{-i \vec{b} \cdot \vec{p}_{t}^{H}} e^{-S_{\mathrm{NLL}}},
\end{aligned}
$$

where the radiator $S_{\mathrm{NLL}}$ reads [32]

$$
S_{\mathrm{NLL}}=-\int[d k] M^{2}(k)\left[e^{i \vec{b} \cdot \vec{k}_{t}} \Theta\left(p_{t}^{J, v}-k_{t}\right)-1\right] .
$$

To evaluate the above integral, we can perform the integration over the rapidity of the radiation $k$ and obtain

$$
\int[d k] M^{2}(k)=\int \frac{d k_{t}}{k_{t}} \frac{d \phi}{2 \pi} R_{\mathrm{NLL}}^{\prime}\left(k_{t}\right),
$$

with

$$
R_{\mathrm{NLL}}^{\prime}\left(k_{t}\right)=4\left(\frac{\alpha_{s}^{\mathrm{CMW}}\left(k_{t}\right)}{\pi} C_{A} \ln \frac{m_{H}}{k_{t}}-\alpha_{s}\left(k_{t}\right) \beta_{0}\right),
$$

where $\beta_{0}$ is the first coefficient of the QCD beta function. The coupling in the CMW scheme is defined as [56-58] $\alpha_{s}^{\mathrm{CMW}}\left(k_{t}\right)=\alpha_{s}\left(k_{t}\right)\left\{1+\left[\left(\alpha_{s}\left(k_{t}\right)\right) / 2 \pi\right][([67 / 18]-\right.$ $\left.\left.\left.\left[\pi^{2} / 6\right]\right) C_{A}-(5 / 9) n_{f}\right]\right\}$, and includes the contribution of nonplanar soft radiation necessary for NLL accuracy in processes with two hard emitters. The azimuthal integral of Eq. (4) leads to

$$
\begin{aligned}
S_{\mathrm{NLL}}= & -\int_{0}^{m_{H}} \frac{d k_{t}}{k_{t}} R_{\mathrm{NLL}}^{\prime}\left(k_{t}\right)\left[J_{0}\left(b k_{t}\right)-1\right] \\
& +\int_{0}^{m_{H}} \frac{d k_{t}}{k_{t}} R_{\mathrm{NLL}}^{\prime}\left(k_{t}\right) J_{0}\left(b k_{t}\right) \Theta\left(k_{t}-p_{t}^{J, v}\right) .
\end{aligned}
$$

In the first integral, we exploit the large- $b$ property $[29,34]$

$$
J_{0}\left(b k_{t}\right) \simeq 1-\Theta\left(k_{t}-b_{0} / b\right)+\mathcal{O}\left(\mathrm{N}^{3} \mathrm{LL}\right),
$$

with $b_{0}=2 e^{-\gamma_{E}}$, to recast Eq. (6) as 


$$
\begin{aligned}
S_{\mathrm{NLL}}= & -L g_{1}\left(\alpha_{s} L\right)-g_{2}\left(\alpha_{s} L\right) \\
& +\int_{0}^{m_{H}} \frac{d k_{t}}{k_{t}} R_{\mathrm{NLL}}^{\prime}\left(k_{t}\right) J_{0}\left(b k_{t}\right) \Theta\left(k_{t}-p_{t}^{J, v}\right),
\end{aligned}
$$

where $\alpha_{s} \equiv \alpha_{s}\left(\mu_{R}\right)$ (with $\mu_{R}$ being the renormalization scale), $L=\ln \left(m_{H} b / b_{0}\right)$, and the $g_{i}$ functions are those used in the standard $p_{t}^{H}$ resummation [59].

The procedure that led to Eq. (3) can be used to extend the above result to higher logarithmic orders. The crucial observation is that, as already stressed, in impact-parameter space the measurement function for $p_{t}^{H}$ is entirely factorized, resulting in a phase factor $e^{i \vec{b} \cdot \vec{k}_{t}}$ for each emission $k$. This implies that the jet-veto constraint $\Theta\left(p_{t}^{J, v}-p_{t}^{J}\right)$ can be included by implementing the jet-veto resummation [34] at the level of the $b$-space integrand, namely, directly in impact-parameter space. We note incidentally that this observation can be applied to the resummation of other pairs of observables for which the measurement function can be factorized.

We now derive the NNLL result. Starting from Eq. (3), the first step is to promote the $R_{\mathrm{NLL}}^{\prime}\left(k_{t}\right)$ function that appears in the radiator $S_{\mathrm{NLL}}$ to NNLL. The corresponding expression is given in Refs. [29,34], and leads to

$$
\begin{aligned}
S_{\mathrm{NNLL}} \equiv & -L g_{1}\left(\alpha_{s} L\right)-g_{2}\left(\alpha_{s} L\right)-\frac{\alpha_{s}}{\pi} g_{3}\left(\alpha_{s} L\right) \\
& +\int_{0}^{m_{H}} \frac{d k_{t}}{k_{t}} R_{\mathrm{NNLL}}^{\prime}\left(k_{t}\right) J_{0}\left(b k_{t}\right) \Theta\left(k_{t}-p_{t}^{J, v}\right) .
\end{aligned}
$$

The above step assumes that the veto on the radiation is encoded in a phase-space constraint of the type (2). While this approximation is correct at NLL, where the jet algorithm does not recombine the emissions with one another, it fails beyond this order. Specifically, up to NNLL, at most two soft emissions can become close in angle (three unordered soft emissions only contribute to $\mathrm{N}^{3} \mathrm{LL}$ ), and therefore may get clustered into the same jet (whose momentum is defined according to the so-called $E$ scheme, where the four momenta of the constituents are added together). The configurations in which the resulting cluster is the leading jet are not correctly described by the constraint in Eq. (2). In order to account for this effect, one has to include a clustering correction [34] in impact parameter space, that reads

$$
\begin{aligned}
\mathcal{F}_{\text {clust }}= & \frac{1}{2 !} \int\left[d k_{a}\right]\left[d k_{b}\right] M^{2}\left(k_{a}\right) M^{2}\left(k_{b}\right) J_{a b}(R) e^{i \vec{b} \cdot \vec{k}_{t, a b}} \\
& \times\left[\Theta\left(p_{t}^{J, v}-k_{t, a b}\right)-\Theta\left(p_{t}^{J, v}-\max \left\{k_{t, a}, k_{t, b}\right\}\right)\right],
\end{aligned}
$$

where $\vec{k}_{t, a b}=\vec{k}_{t, a}+\vec{k}_{t, b}$ and $k_{t, a b}$ is its magnitude. The constraint $J_{a b}(R)=\Theta\left(R^{2}-\Delta \eta_{a b}^{2}-\Delta \phi_{a b}^{2}\right)$ restricts the phase space to the region where the recombination between the two emissions takes place. Here $R$ is the jet radius and $\Delta \eta_{a b}$ and $\Delta \phi_{a b}$ are the pseudorapidity and azimuthal separation between the two emissions, respectively. We observe that Eq. (10) differs from the corresponding clustering correction for the standard jet-veto resummation [34] by the factor $e^{i \vec{b} \cdot \vec{k}_{t, a b}}$, which accounts for the $p_{t}^{H}$ constraint in impact-parameter space.

Equation (10) describes the clustering correction due to two independent soft emissions. A similar correction arises when the two soft emissions $k_{a}, k_{b}$ are correlated, i.e., their squared matrix element cannot be factorized into the product of two independent squared amplitudes. The contribution of a pair of correlated emissions is accounted for in the CMW scheme for the strong coupling that was already used in the NLL radiator (4). However, such a scheme is obtained by integrating inclusively over the correlated squared amplitude $\tilde{M}^{2}\left(k_{a}, k_{b}\right)$, given in Ref. [63]. While this inclusive treatment is accurate at NLL, at NNLL one needs to correct for configurations in which the two correlated emissions are not clustered together by the jet algorithm. This amounts to including a correlated correction [34] of the form

$$
\begin{aligned}
\mathcal{F}_{\text {correl }}= & \frac{1}{2 !} \int\left[d k_{a}\right]\left[d k_{b}\right] \tilde{M}^{2}\left(k_{a}, k_{b}\right)\left[1-J_{a b}(R)\right] e^{i \vec{b} \cdot \vec{k}_{t, a b}} \\
& \times\left[\Theta\left(p_{t}^{J, v}-\max \left\{k_{t, a}, k_{t, b}\right\}\right)-\Theta\left(p_{t}^{J, v}-k_{t, a b}\right)\right] .
\end{aligned}
$$

The corrections (10) and (11) describe the aforementioned effects for a single pair of emissions. At NNLL, all remaining emissions can be considered to be far in angle from the pair $k_{a}, k_{b}$, and therefore they never get clustered with the jets resulting from Eqs. (10) and (11).

As a final step towards a NNLL prediction, one must account for non-soft collinear emissions off the initial-state particles. Since a $k_{t}$-type jet algorithm never clusters the soft emissions discussed above with non-soft collinear radiation, the latter can be conveniently handled by taking a Mellin transform of the resummed cross section. In Mellin space, the collinear radiation gives rise to the scale evolution of the parton densities $f(\mu)$ and of the collinear coefficient functions $C\left(\alpha_{s}\right)$. The latter, as well as the hardvirtual corrections $\mathcal{H}\left(\alpha_{s}\right)$, must be included at the one-loop level for a NNLL resummation. The equivalent of the clustering and correlated corrections for hard-collinear radiation enters only at $\mathrm{N}^{3} \mathrm{LL}$, and therefore is neglected in the following.

After applying to hard-collinear emissions the same procedure detailed above for soft radiation, we obtain the main result of this Letter, namely, the NNLL master formula for the $p_{t}^{H}$ spectrum with a jet veto $p_{t}^{J, v}$, differential in the Higgs rapidity $y^{H}$ : 


$$
\begin{aligned}
& \frac{d \sigma\left(p_{t}^{J, v}\right)}{d y^{H} d^{2} \vec{p}_{t}^{H}}=\frac{2 \pi}{s} M_{\mathrm{g} \rightarrow \mathrm{H}}^{2} \mathcal{H}\left(\alpha_{s}\left(m_{H}\right)\right) \int_{\mathcal{C}_{1}} \frac{d \nu_{1}}{2 \pi i} \int_{\mathcal{C}_{2}} \frac{d \nu_{2}}{2 \pi i} x_{1}^{-\nu_{1}} x_{2}^{-\nu_{2}} \int \frac{d^{2} \vec{b}}{4 \pi^{2}} e^{-i \vec{b} \cdot \vec{p}_{t}^{H}} e^{-S_{\mathrm{NNLL}}}\left(1+\mathcal{F}_{\text {clust }}+\mathcal{F}_{\text {correl }}\right) \\
& \times\left[\mathcal{P} e^{\int_{0}^{m_{H}} \frac{d \mu}{\mu} \Gamma_{\nu_{1}}\left(\alpha_{s}(\mu)\right)\left(\Theta\left(p_{t}^{J, v}-\mu\right) J_{0}(b \mu)-1\right)}\right]_{c_{1} a_{1}}\left[\mathcal{P} e^{\int_{0}^{m_{H}} \frac{d \mu}{\mu} \Gamma_{\nu_{2}}\left(\alpha_{s}(\mu)\right)\left(\Theta\left(p_{t}^{j, v}-\mu\right) J_{0}(b \mu)-1\right)}\right]_{c_{2} a_{2}} f_{\nu_{1}, a_{1}}\left(m_{H}\right) f_{\nu_{2}, a_{2}}\left(m_{H}\right) \\
& \times e^{\int_{0}^{m_{H}} \frac{d \mu}{\mu}\left[\Gamma_{\nu_{1}}^{(C)}\left(\alpha_{s}(\mu)\right)\right]_{g c_{1}}\left(\Theta\left(p_{t}^{J, v}-\mu\right) J_{0}(b \mu)-1\right)} e^{\int_{0}^{m_{H}} \frac{d \mu}{\mu}\left[\Gamma_{\nu_{2}}^{(C)}\left(\alpha_{s}(\mu)\right)\right]_{g c_{2}}\left(\Theta\left(p_{t}^{J, v}-\mu\right) J_{0}(b \mu)-1\right)} C_{\nu_{1}, g c_{1}}\left(\alpha_{s}\left(m_{H}\right)\right) C_{\nu_{2}, g c_{2}}\left(\alpha_{S}\left(m_{H}\right)\right),
\end{aligned}
$$

where $x_{1,2}=m_{H} / \sqrt{s} e^{ \pm y^{H}}$, and $M_{\mathrm{gg} \rightarrow H}^{2}$ is the Born squared matrix element including the partonic flux factor. The $\nu_{\ell}$ subscripts denote the Mellin transform, while the latin letters represent flavor indices, and the sum over repeated indices is understood. Here $\boldsymbol{\Gamma}_{\nu_{e}}$ and $\boldsymbol{\Gamma}_{\nu_{\ell}}^{(C)}$ are the anomalous dimensions describing the scale evolution of the parton densities and coefficient functions, respectively. The contours $\mathcal{C}_{1}$ and $\mathcal{C}_{2}$ lie parallel to the imaginary axis to the right of all singularities of the integrand. The (anti)path-ordering symbol $\mathcal{P}$ has a formal meaning, and encodes the fact that the evolution operators are matrices in flavor space. All the ingredients of Eq. (12) are given in Ref. [59]. The multidifferential distribution $d \sigma / d p_{t}^{J} d y^{H} d^{2} \vec{p}_{t}^{H}$ is simply obtained by taking the derivative of Eq. (12) in $p_{t}^{J, v}$.

All integrals entering the above formula are finite in four dimensions and can be evaluated numerically to very high precision. We point out that, similarly to the standard $p_{t}^{H}$ resummation [28,29], the result in Eq. (12) can also be deduced directly in momentum space, without resorting to an impact-parameter formulation. The momentum-space approach is particularly convenient for computational purposes, in that it gives access to differential information on the QCD radiation, thereby enabling an efficient Monte Carlo calculation. Therefore, we adopt the latter method for a practical implementation of Eq. (12). The relevant formulas are detailed in Ref. [59], and implemented in the RadisH program.

For the numerical results presented below, we choose $\sqrt{s}=13 \mathrm{TeV}$ and we adopt the NNPDF3.1 set [64] of parton densities (PDFs) at NNLO, with $\alpha_{s}\left(M_{Z}\right)=0.118$. The evolution of the PDFs is performed with the LHAPDF [65] package and all convolutions are handled with HOPPET [66]. We set the renormalization and factorization scale to $\mu_{R}=\mu_{F}=m_{H}=125 \mathrm{GeV}$, and $R=0.4$. Figure 1 shows Eq. (12) integrated over the rapidity of the Higgs boson $y^{H}$ and over the $\vec{p}_{t}^{H}$ azimuth, as a function of $p_{t}^{H}$ and $p_{t}^{J, v}$. We observe the typical peaked structure along the $p_{t}^{H}$ direction, as well as the Sudakov suppression at small $p_{t}^{J, v}$. The two-dimensional distribution also features a Sudakov shoulder along the diagonal $p_{t}^{H} \sim p_{t}^{J, v}$, which originates from the sensitivity of the differential spectrum to soft radiation in this region beyond leading order [67]. Equation (12) provides a resummation of the logarithms associated with the shoulder in the regime $p_{t}^{H} \sim p_{t}^{J, v} \ll m_{H}$, which can be appreciated by the absence of an integrable singularity in this region.
To verify the correctness of Eq. (12), we perform a number of checks. As a first observation, we note that in the region $p_{t}^{J, v} \gtrsim m_{H}$, the terms $\mathcal{F}_{\text {clust }}$ and $\mathcal{F}_{\text {correl }}$ vanish by construction and, as expected, one recovers the NNLL resummation for the inclusive $p_{t}^{H}$ spectrum. Conversely, considering the limit $p_{t}^{H} \gtrsim m_{H}$ (i.e., small $b$ ), Eq. (12) reproduces the standard NNLL jet-veto resummation of Ref. [34] as detailed in Ref. [59]. As a further test, we expand Eq. (12) to second order in $\alpha_{s}$ relative to the Born, and compare the result with an $\mathcal{O}\left(\alpha_{s}^{2}\right)$ fixed-order calculation for the inclusive production of a Higgs boson plus one jet [68-70], with jets defined according to the anti- $k_{t}$ algorithm [55]. In particular, to avoid the perturbative instability associated with the Sudakov shoulder, we calculate the double cumulant

$$
\sigma\left(p_{t}^{J, v}, p_{t}^{H, v}\right) \equiv \int d y^{H} d^{2} \vec{p}_{t}^{H} \frac{d \sigma\left(p_{t}^{J, v}\right)}{d y^{H} d^{2} \vec{p}_{t}^{H}} \Theta\left(p_{t}^{H, v}-\left|\vec{p}_{t}^{H}\right|\right),
$$

and define the quantity

$$
\Delta\left(p_{t}^{J, v}, p_{t}^{H, v}\right)=\sigma^{\mathrm{NNLO}}\left(p_{t}^{J, v}, p_{t}^{H, v}\right)-\sigma_{\exp }^{\mathrm{NNLL}}\left(p_{t}^{J, v}, p_{t}^{H, v}\right),
$$

where $\sigma^{\mathrm{NNLO}}\left(p_{t}^{J, v}, p_{t}^{H, v}\right)$ is computed by taking the difference between the NNLO total Higgs-production cross section [71-73], obtained with the ggHiggs program [74], and the NLO Higgs + jet cross section for

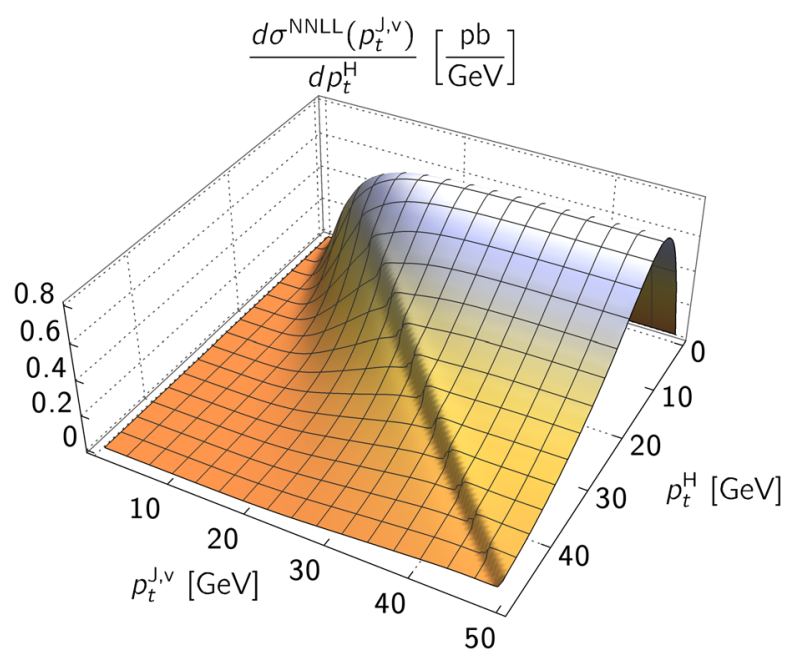

FIG. 1. The NNLL differential distribution (12), integrated over the Higgs-boson rapidity $y^{H}$ and over the $\vec{p}_{t}^{H}$ azimuth, as a function of $p_{t}^{H}$ and $p_{t}^{J, v}$. 


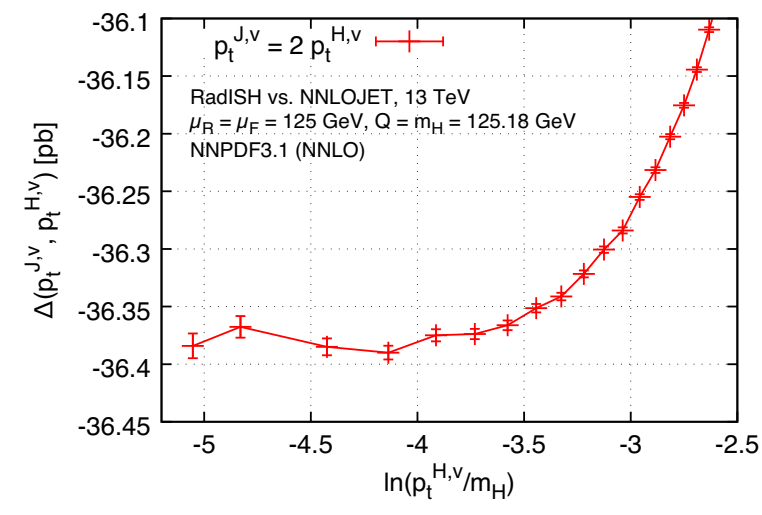

FIG. 2. $\Delta\left(p_{t}^{J, v}, p_{t}^{H, v}\right)$, as defined in the text, at second order in $\alpha_{s}$ as a function of $\ln \left(p_{t}^{H, v} / m_{H}\right)$, for $p_{t}^{J, v}=2 p_{t}^{H, v}$. This test features a slightly different Higgs mass, $m_{H}=125.18 \mathrm{GeV}$.

$\left(p_{t}^{J}>p_{t}^{J, v}\right) \vee\left(p_{t}^{H}>p_{t}^{H, v}\right)$, calculated with the NNLOJET program [18]. Given that the NNLL prediction controls all divergent terms at the second perturbative order, one expects the quantity $\Delta$ to approach a constant value of $\mathrm{N}^{3} \mathrm{LL}$ nature in the $p_{t}^{H} \rightarrow 0$ limit. Figure 2 displays this limit for $p_{t}^{J, v}=2 p_{t}^{H, v}$, which shows an excellent convergence towards a constant, thereby providing a robust test of Eq. (12).

As a phenomenological application of our result, we set $p_{t}^{J, v}=30 \mathrm{GeV}$ in accordance with the LHC experiments. While Eq. (12) provides an accurate description of the spectrum in the small- $p_{t}^{H}$ region, in order to reliably extend the prediction to larger $p_{t}^{H}$ values one needs to match the resummed formula to a fixed-order calculation, in which the hard radiation is correctly accounted for. We thus match the NNLL result to the NLO Higgs + jet $p_{t}^{H}$ distribution obtained with the program MCFM-8.3 [75,76] by means of the multiplicative matching formulated in Refs. [23,31,77]. We adopt the setup outlined above, and in addition we introduce the resummation scale $Q$ as detailed in the Supplemental Material [59] as a mean to assess the uncertainties due to missing higher logarithmic corrections. To estimate the theoretical uncertainty of our final prediction, we perform a variation of the renormalization and factorization scales by a factor of 2 about the central value $\mu_{R}=\mu_{F}=m_{H}$, while keeping $1 / 2 \leq \mu_{R} / \mu_{F} \leq 2$. Moreover, for central $\mu_{R}$ and $\mu_{F}$ scales, we vary the resummation scale by a factor of 2 around $Q=m_{H} / 2$, and take the envelope of all the above variations. Figure 3 compares the NNLL + NLO prediction to the NLL + LO, and to the fixed-order NLO result. The integral of the NNLL + NLO (NLL + LO) distribution yields the corresponding jet-vetoed cross section at NNLL + NNLO (NLL + NLO) [34].

We observe a good perturbative convergence for the resummed predictions to the left of the peak, where logarithmic corrections dominate. Above $p_{t}^{H} \sim 10 \mathrm{GeV}$, the NNLL + NLO prediction differs from the NLL + LO

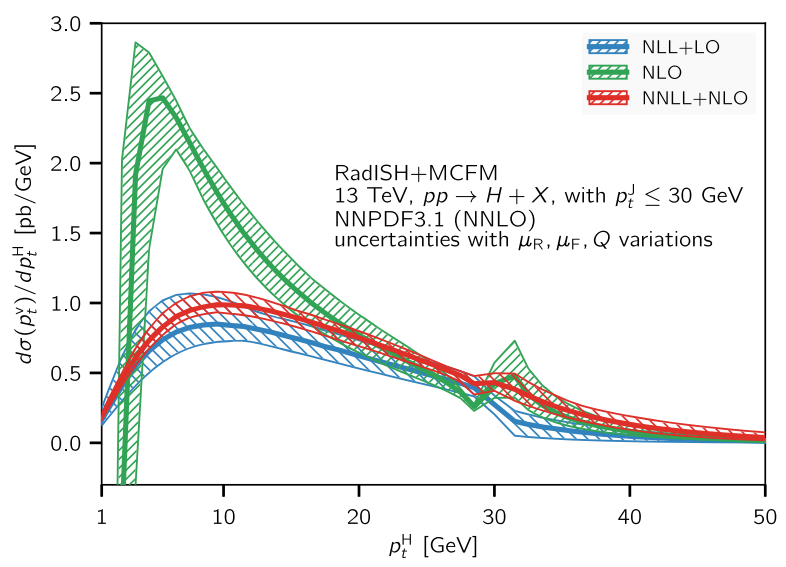

FIG. 3. Matched NNLL + NLO (red band), NLL + LO (blue band), and fixed-order NLO (green band) $p_{t}^{H}$ differential distributions for $p_{t}^{J, v}=30 \mathrm{GeV}$, with theoretical uncertainties estimated as explained in the main text.

due to the large NLO $K$ factor in the considered process. The residual perturbative uncertainty in the NNLL + NLO distribution is of $\mathcal{O}(10 \%)$ for $p_{t}^{H} \lesssim p_{t}^{J, v}$. The comparison to the NLO fixed order shows the importance of resummation across the whole $p_{t}^{H}$ region, and a much reduced sensitivity to the Sudakov shoulder at $p_{t}^{H} \sim p_{t}^{J, v}$ (in Fig. 3 we use a $2 \mathrm{GeV}$ bin across the shoulder).

In this Letter we have formulated the first doubledifferential resummation for an observable defined through a jet algorithm in hadronic collisions. As a case study, we considered the production of a Higgs boson in gluon fusion with transverse momentum $p_{t}^{H}$ in association with jets satisfying the veto requirement $p_{t}^{J} \leq p_{t}^{J, v}$. In the limit $p_{t}^{H}, p_{t}^{J, v} \ll m_{H}$, we performed the resummation of the large logarithms $\ln \left(m_{H} / p_{t}^{H}\right), \ln \left(m_{H} / p_{t}^{J, v}\right)$ up to NNLL, resulting in an accurate theoretical prediction for this physical observable. As a phenomenological application, we presented matched NNLL + NLO results at the LHC. Our formulation can be applied to the production of any colorsinglet system, and it is relevant in a number of phenomenological applications that will be explored in future work.

We would like to thank Andrea Banfi and Gavin Salam for stimulating discussions on the subject of this Letter, and Emanuele Re and Giulia Zanderighi for constructive comments on the manuscript. We are very grateful to Alexander Huss for kindly providing us with a cross check of our results with the NNLOJET program. The work of P. M. has been supported by the Marie Skłodowska Curie Individual Fellowship Contract No. 702610 Resummation4PS. L. R. is supported by the ERC Starting Grant REINVENT (714788), and acknowledges the CERN Theoretical Physics Department for hospitality and support during part of this work, and the CINECA award under the ISCRA initiative for the availability of the high-performance computing resources needed for this work. 
[1] G. Aad et al. (ATLAS Collaboration), Phys. Lett. B 716, 1 (2012).

[2] S. Chatrchyan et al. (CMS Collaboration), Phys. Lett. B 716, 30 (2012).

[3] M. Cepeda et al. (HL/HE WG2 Group), CERN Yellow Rep. Monogr. 7, 221 (2019).

[4] F. Bishara, U. Haisch, P. F. Monni, and E. Re, Phys. Rev. Lett. 118, 121801 (2017).

[5] Y. Soreq, H. X. Zhu, and J. Zupan, J. High Energy Phys. 12 (2016) 045.

[6] A. Banfi, A. Martin, and V. Sanz, J. High Energy Phys. 08 (2014) 053.

[7] C. Grojean, E. Salvioni, M. Schlaffer, and A. Weiler, J. High Energy Phys. 05 (2014) 022.

[8] M. Schlaffer, M. Spannowsky, M. Takeuchi, A. Weiler, and C. Wymant, Eur. Phys. J. C 74, 3120 (2014).

[9] M. Grazzini, A. Ilnicka, M. Spira, and M. Wiesemann, J. High Energy Phys. 03 (2017) 115.

[10] A. Banfi, A. Bond, A. Martin, and V. Sanz, J. High Energy Phys. 11 (2018) 171.

[11] A. Banfi, B. M. Dillon, W. Ketaiam, and S. Kvedaraite, J. High Energy Phys. 01 (2020) 089.

[12] M. Aaboud et al. (ATLAS Collaboration), Phys. Lett. B 789, 508 (2019).

[13] A. M. Sirunyan et al. (CMS Collaboration), Phys. Lett. B 791, 96 (2019).

[14] M. Aaboud et al. (ATLAS Collaboration), Phys. Rev. D 98 , 052005 (2018).

[15] R. Boughezal, F. Caola, K. Melnikov, F. Petriello, and M. Schulze, Phys. Rev. Lett. 115, 082003 (2015).

[16] R. Boughezal, C. Focke, W. Giele, X. Liu, and F. Petriello, Phys. Lett. B 748, 5 (2015).

[17] F. Caola, K. Melnikov, and M. Schulze, Phys. Rev. D 92 , 074032 (2015).

[18] X. Chen, J. Cruz-Martinez, T. Gehrmann, E. W. N. Glover, and M. Jaquier, J. High Energy Phys. 10 (2016) 066.

[19] J. M. Campbell, R. K. Ellis, and S. Seth, J. High Energy Phys. 10 (2019) 136.

[20] J. M. Lindert, K. Melnikov, L. Tancredi, and C. Wever, Phys. Rev. Lett. 118, 252002 (2017).

[21] J. M. Lindert, K. Kudashkin, K. Melnikov, and C. Wever, Phys. Lett. B 782, 210 (2018).

[22] S. P. Jones, M. Kerner, and G. Luisoni, Phys. Rev. Lett. 120, 162001 (2018).

[23] F. Caola, J. M. Lindert, K. Melnikov, P. F. Monni, L. Tancredi, and C. Wever, J. High Energy Phys. 09 (2018) 035.

[24] T. Neumann, J. Phys. Commun. 2, 095017 (2018).

[25] G. Bozzi, S. Catani, D. de Florian, and M. Grazzini, Nucl. Phys. B737, 73 (2006).

[26] T. Becher, M. Neubert, and D. Wilhelm, J. High Energy Phys. 05 (2013) 110.

[27] D. Neill, I. Z. Rothstein, and V. Vaidya, J. High Energy Phys. 12 (2015) 097.

[28] P. F. Monni, E. Re, and P. Torrielli, Phys. Rev. Lett. 116, 242001 (2016).

[29] W. Bizoń, P. F. Monni, E. Re, L. Rottoli, and P. Torrielli, J. High Energy Phys. 02 (2018) 108.

[30] X. Chen, T. Gehrmann, N. Glover, A. Huss, Y. Li, D. Neill, M. Schulze, I. W. Stewart, and H. X. Zhu, Phys. Lett. B 788, 425 (2019).
[31] W. Bizoń, X. Chen, A. Gehrmann-De Ridder, T. Gehrmann, N. Glover, A. Huss, P. F. Monni, E. Re, L. Rottoli, and P. Torrielli, J. High Energy Phys. 12 (2018) 132.

[32] A. Banfi, G. P. Salam, and G. Zanderighi, J. High Energy Phys. 06 (2012) 159.

[33] T. Becher and M. Neubert, J. High Energy Phys. 07 (2012) 108.

[34] A. Banfi, P. F. Monni, G. P. Salam, and G. Zanderighi, Phys. Rev. Lett. 109, 202001 (2012).

[35] T. Becher, M. Neubert, and L. Rothen, J. High Energy Phys. 10 (2013) 125.

[36] I. W. Stewart, F. J. Tackmann, J. R. Walsh, and S. Zuberi, Phys. Rev. D 89, 054001 (2014).

[37] A. Banfi, P. F. Monni, and G. Zanderighi, J. High Energy Phys. 01 (2014) 097.

[38] J. K. L. Michel, P. Pietrulewicz, and F. J. Tackmann, J. High Energy Phys. 04 (2019) 142.

[39] A. Banfi, F. Caola, F. A. Dreyer, P. F. Monni, G. P. Salam, G. Zanderighi, and F. Dulat, J. High Energy Phys. 04 (2016) 049.

[40] P. Sun, C.-P. Yuan, and F. Yuan, Phys. Rev. Lett. 114, 202001 (2015).

[41] Y. T. Chien, D. Y. Shao, and B. Wu, J. High Energy Phys. 11 (2019) 025.

[42] P. Sun, J. Isaacson, C.-P. Yuan, and F. Yuan, Phys. Lett. B 769, 57 (2017).

[43] A. J. Larkoski, I. Moult, and D. Neill, J. High Energy Phys. 09 (2014) 046.

[44] M. Procura, W. J. Waalewijn, and L. Zeune, J. High Energy Phys. 10 (2018) 098.

[45] G. Lustermans, J. K. L. Michel, F. J. Tackmann, and W. J. Waalewijn, J. High Energy Phys. 03 (2019) 124.

[46] S. Marzani, Phys. Rev. D 93, 054047 (2016).

[47] S. Forte and C. Muselli, J. High Energy Phys. 03 (2016) 122.

[48] E. Laenen, G. F. Sterman, and W. Vogelsang, Phys. Rev. D 63, 114018 (2001).

[49] A. Kulesza, G. F. Sterman, and W. Vogelsang, Phys. Rev. D 69, 014012 (2004).

[50] G. Lustermans, W. J. Waalewijn, and L. Zeune, Phys. Lett. B 762, 447 (2016).

[51] C. Muselli, S. Forte, and G. Ridolfi, J. High Energy Phys. 03 (2017) 106.

[52] M. Bonvini and S. Marzani, Phys. Rev. Lett. 120, 202003 (2018).

[53] G. Parisi and R. Petronzio, Nucl. Phys. B154, 427 (1979).

[54] J. C. Collins, D. E. Soper, and G. F. Sterman, Nucl. Phys. B250, 199 (1985).

[55] M. Cacciari, G. P. Salam, and G. Soyez, J. High Energy Phys. 04 (2008) 063.

[56] S. Catani, B. R. Webber, and G. Marchesini, Nucl. Phys. B349, 635 (1991).

[57] A. Banfi, B. K. El-Menoufi, and P. F. Monni, J. High Energy Phys. 01 (2019) 083.

[58] S. Catani, D. De Florian, and M. Grazzini, Eur. Phys. J. C 79, 685 (2019).

[59] See Supplemental Material at http://link.aps.org/ supplemental/10.1103/PhysRevLett.124.252001 for additional details on the results of the Letter, including Refs. [60-62]. 
[60] D. de Florian and M. Grazzini, Nucl. Phys. B616, 247 (2001).

[61] T. Becher and M. Neubert, Eur. Phys. J. C 71, 1665 (2011).

[62] R. K. Ellis, W. J. Stirling, and B. R. Webber, QCD and Collider Physics (Cambridge University Press, Cambridge, England, 1996), https://doi.org/10.1017/CBO9780511628788.

[63] Y. L. Dokshitzer, A. Lucenti, G. Marchesini, and G. P. Salam, Nucl. Phys. B511 (1998) 396; B593, 729 (2001).

[64] R. D. Ball et al. (NNPDF Collaboration), Eur. Phys. J. C 77, 663 (2017).

[65] A. Buckley, J. Ferrando, S. Lloyd, K. Nordström, B. Page, M. Rüfenacht, M. Schönherr, and G. Watt, Eur. Phys. J. C 75, 132 (2015).

[66] G. P. Salam and J. Rojo, Comput. Phys. Commun. 180, 120 (2009).

[67] S. Catani and B. R. Webber, J. High Energy Phys. 10 (1997) 005 .

[68] D. de Florian, M. Grazzini, and Z. Kunszt, Phys. Rev. Lett. 82, 5209 (1999).

[69] V. Ravindran, J. Smith, and W. L. Van Neerven, Nucl. Phys. B634, 247 (2002).
[70] C. J. Glosser and C. R. Schmidt, J. High Energy Phys. 12 (2002) 016.

[71] R. V. Harlander and W. B. Kilgore, Phys. Rev. Lett. 88, 201801 (2002).

[72] C. Anastasiou and K. Melnikov, Nucl. Phys. B646, 220 (2002).

[73] V. Ravindran, J. Smith, and W. L. van Neerven, Nucl. Phys. B665, 325 (2003).

[74] R. D. Ball, M. Bonvini, S. Forte, S. Marzani, and G. Ridolfi, Nucl. Phys. B874, 746 (2013).

[75] J. M. Campbell, R. K. Ellis, and W. T. Giele, Eur. Phys. J. C 75, 246 (2015).

[76] R. Boughezal, J. M. Campbell, R. K. Ellis, C. Focke, W. Giele, X. Liu, F. Petriello, and C. Williams, Eur. Phys. J. C 77, 7 (2017).

[77] W. Bizoń, A. Gehrmann-De Ridder, T. Gehrmann, N. Glover, A. Huss, P. F. Monni, E. Re, L. Rottoli, and D. Walker, arXiv:1905.05171.

Correction: Equation (12) and related text contained errors and have been fixed. 